\title{
INTENSIVE GLUCOSE CONTROL IN THE TREATMENT OF HYPERGLYCEMIA IN PATIENTS WITH AN ACUTE EXACERBATION OF CHRONIC OBSTRUCTIVE PULMONARY DISEASE: A PROSPECTIVE STUDY
}

\author{
L. Gao ${ }^{1}, H . \mathrm{Ren}^{1}, \mathrm{Q} . \mathrm{Hao}{ }^{2}$
}

\begin{abstract}
Background and Objective: This study was conducted to evaluate the influence of IGC in the treatment of hyperglycemia combined with AECOPD. Methods: A total of 267 consecutively hospitalized patients with AECOPD combined with hyperglycemia in the general wards of the East Ward of Sichuan Academy of Medical Sciences \& Sichuan Provincial People's Hospital and West China Hospital from May 2007 to August 2013 were included in this study. All the patients were allocated to either the IGC group or the control group according to the doctor's recommendation. The short-term mortality rate as well as the duration and medical cost of the hospitalization were investigated. Results: The median length of the hospital stay was 15 days in the IGC group, whereas the median was 12 days $(\mathrm{p}=0.05)$ in the control group. The median medical cost was 8,866 Yuan in the IGC group, whereas this value was 8,051 Yuan in the control group. However, the medical costs, the mortality rates and the positive sputum culture rates were not statistically different between the two groups. There were more cases of hypoglycemia and hypokalemia in the IGC group than in the control group. Conclusions: Intensive glucose control treatment did not result in any benefits in the patients with hyperglycemia and AECOPD and may prolong the length of stay and increase the risk of hypoglycemia and hypokalemia.
\end{abstract}

Key words: Acute exacerbation, chronic obstructive pulmonary disease, hyperglycemia; intensive glucose control, length of stay.

Abbreviations: AECOPD: Acute exacerbation of chronic obstructive pulmonary disease; CBG: Capillary blood glucose; COPD: Chronic obstructive pulmonary disease; FBG: Fasting blood glucose; IGC: Intensive glucose control; PBG: Post-prandial blood glucose.

\section{Introduction}

Chronic obstructive pulmonary disease (COPD) is the fourth most common cause of death in the world and will become the third most common in a few years. Acute exacerbation is a frequent event in the natural course of COPD (AECOPD) and is characterized by the acute aggravation of respiratory symptoms such as dyspnea and coughing with or without sputum that require specific treatment regimens (1). One of the earliest and most quoted definitions was put forth by Anthonisen (2) and is based on an increase in the symptoms of dyspnea, sputum volume and sputum purulence, with or without symptoms of an upper respiratory tract infection, and

1. East Ward of Sichuan Academy of Medical Sciences \& Sichuan Provincial People's Hospital; 2. West China Hospital, Sichuan University.

Corresponding Author: Hui Ren Damianhonghe North Road No. 585, Longquanyi district, East Ward of Sichuan Academy of Medical Sciences \& Sichuan Provincial People's Hospital, Chengdu, Sichuan province, China (ZIP: 610000). Tel: +86-02888424442; Cell-phone: +86-13688339617; Fax: +86-028-88424444; E-mail: 592897298@qq.com

Received July 1, 2014

Accepted for publication August 21, 2014 then subdivided depending on the number of symptoms present. The AECOPD episodes vary in severity from mild exacerbations, which can normally be managed by the patient at home, to moderate exacerbations, which require consultation with primary care physicians, and severe exacerbations, which require hospitalization. AECOPD may occur during the treatment period. AECOPD increases the overall mortality rate of patients and has a serious impact on the quality of life.

Approximately $50 \%$ of all the patients with an exacerbation of COPD admitted to the hospital have an elevated random blood glucose level of $>7 \mathrm{mmol} / \mathrm{l}$ (3), and the care of these patients is often complicated by hypoxia, acidosis and systemic inflammation, which includes the induction of peripheral insulin resistance. In AECOPD patients, hyperglycemia is associated with an increased risk of hospital-acquired pulmonary infections and increased odds of having positive sputum cultures $(4,5)$. Experimental studies also reveal that hyperglycemia is positively correlated with the plasma 
levels of cytokines, including IL-18, IL-6 and TNF-a (6). High glucose concentrations can also contribute to muscle wasting (7). However, there was no causative link demonstrated between hyperglycemia and poor outcomes of AECOPD patients.

In the patients who have a lung infection and stroke, IGC seems to have more benefit than risk according to previous studies $(8,9)$. However, the evidence in patients with AECOPD complicated by hyperglycemia is very conflicting. Furthermore, most of the evidence has been from studies conducted in ICUs and retrospective studies. To the best of our knowledge, there are very few prospective trials that focus on the management of acute hyperglycemia in AECOPD patients in the general wards. The current best practice is based on the judgment and clinical experience of physicians. Therefore, we conducted a prospective study to observe the effects of intensive glucose control in patients with AECOPD combined with hyperglycemia.

\section{Methods}

A prospective, two-arm cohort study was conducted in patients with an exacerbation of COPD admitted to the general wards of the East Ward of Sichuan Academy of Medical Sciences \& Sichuan Provincial People's Hospital and West China Hospital. The study was conducted in accordance with the Declaration of Helsinki and was approved by the ethics committee of Sichuan Academy of Medical Sciences \& Sichuan Provincial People's Hospital.

Patients who were diagnosed with AECOPD according to the published guidelines (10) for COPD and were consecutively admitted to the above two hospitals from May 2007 to August 2013 were enrolled. Hyperglycemia was diagnosed according to the following criteria: fasting blood glucose $(\mathrm{FBG}) \geq 10 \mathrm{mmol} / \mathrm{l}$; 2-hour post-prandial blood glucose $(\mathrm{PBG}) \geq 11.1 \mathrm{mmol} / \mathrm{l} ; 2$-hour post-OGTT blood glucose $\geq 11.1 \mathrm{mmol} / 1$. Only patients with an exacerbation of COPD and hyperglycemia were included in this study.

Individuals with any one of the following conditions were excluded: refusal of IGC or blood glucose testing; ICU admission; type 1 diabetes mellitus; diabetes ketoacidosis or hyperosmolar coma; an admission predicted to be short $(<48 \mathrm{~h})$; moribund, very severe complicated disease (e.g., unstable angina pectoris or acute myocardial infarction; an acute cerebral vascular accident; serious arrhythmia; previous history of myocardial infarction or a cerebral vascular accident; the requirement for invasive mechanical ventilation; a pneumothorax; upper gastrointestinal bleeding) or not undergoing current treatment; an increased risk of hypoglycemia. Written informed consent was obtained from all the included subjects and their statutory agents.

The diagnosis of COPD was based on the opinion of a respiratory specialist or spirometry. Smoking history, the number of days of admission, and the medical cost of hospitalization were recorded. All the subjects received medical care for their exacerbations of COPD at the discretion of the treating clinician according to the COPD guidelines in Chinese.

All the included patients received a capillary blood glucose $(\mathrm{CBG})$ test at the beginning of the study and then four times per day (fasting and post-prandial blood glucose, FBG and PBG). All the blood glucose tests were conducted with fingertip blood using a bedside glucometer (Accu-Chek Performa, Roche, USA) that was calibrated weekly. The subjects in the intensive glucose control group were treated using insulin as required such that the FBG was controlled to $4.4-6.1 \mathrm{mmol} / 1$ and the PBG was $<8 \mathrm{mmol} / \mathrm{l}$. The other subjects constituted the control group. The FBG and PBG levels in the patients in this group were typically less than $11.1 \mathrm{mmol} / 1$.

The primary endpoint in this study was safety, including the following: the frequency of hypoglycemia, defined as agitation (other than mild), drowsiness, confusion, and ataxia that is resolved with the administration of carbohydrate or a $C B G \leq 3.3 \mathrm{mmol} / 1$ or CBG $<2.2 \mathrm{mmol} / 1$ with or without sweating, tremor, palpitations, or tachycardia; and the frequency of hypokalemia, defined as a serum potassium level $<3.5$ $\mathrm{mmol} / \mathrm{l}$.

The length and medical cost of the hospital stay was assessed in this study. The discharge assessments and decisions and all the medical prescriptions for all of the subjects were made by the respiratory medicine teams in the two hospitals; these teams were blinded regarding the group information of the subjects. The length of the hospital stay was calculated according to the record of admission and separation dates. All the expenses related to the medical care for each admission were totaled and considered as the medical cost of the hospital stay.

The baseline data and demographic information for all the subjects included age, gender, smoking status, sputum cultures and the presence of pneumonia. These data were collected to analyze additional variables.

Continuous variables with a normal distribution were expressed as the mean \pm standard deviation (SD) and were compared between the two groups using an unpaired Student's $t$ test. The non-normally distributed variables (e.g., the length and expense of the hospital stay) were expressed as the median (interquartile range, IQR) and were compared between the two groups using the Mann-Whitney U test. The categorical variables were presented as percentages and absolute numbers and were compared between groups using a Chi-square test or Fisher's exact test (where the expected cell count was $<5$ ). All the statistical analyses were performed with the SPSS13.0 software (SPSS Inc., Chicago, IL, USA). A p value $\leq 0.05$ was considered to be statistically significant, and all the $p$ values were two-sided. 


\section{Results}

From May 2007 to August 2013, a total of 267 patients with AECOPD combined with hyperglycemia were ultimately enrolled in this study. The baseline characteristics of the patients in the two groups are shown in Table 1.

Table 1

Baseline data of patients with a comorbidity of AECOPD and hyperglycemia

\begin{tabular}{lccc}
\hline & $\begin{array}{c}\text { Intensive glucose } \\
\text { control group } \\
(\mathbf{n}=\mathbf{6 9 )}\end{array}$ & $\begin{array}{c}\text { Control } \\
\text { group } \\
(\mathbf{n = 1 9 8})\end{array}$ & P value \\
\hline Age (years) & $75.09 \pm 7.14$ & $74.74 \pm 7.62$ & $\mathrm{NS}$ \\
Gender (male/ female) & $60 / 9$ & $153 / 45$ & $\mathrm{NS}$ \\
COPD duration (years) & $20(12.25,20)$ & $20(10,30)$ & $\mathrm{NS}$ \\
Smoking history (yes/no) & $18 / 51$ & $36 / 162$ & $\mathrm{NS}$ \\
Fasting blood glucose (mmol/L) & $7.51 \pm 0.39$ & $7.45 \pm 1.65$ & $\mathrm{NS}$ \\
Post-prandial blood glucose & & & \\
(mmol/L) & $9.77 \pm 0.78$ & $10.90 \pm 2.03$ & $\mathrm{NS}$ \\
Pneumonia (yes/no) & $9 / 60$ & $51 / 147$ & $\mathrm{NS}$ \\
APACHE II score & $15.9 \pm 2.8$ & $17.4 \pm 5.6$ & $\mathrm{NS}$ \\
pH value & $7.37 \pm 0.1$ & $7.36 \pm 0.1$ & $\mathrm{NS}$ \\
pCO2 (mmHg) & $49.6 \pm 11.7$ & $49.2 \pm 16.2$ & $\mathrm{NS}$ \\
pO2 (mmHg) & $70.1 \pm 22.3$ & $72.7 \pm 26.3$ & $\mathrm{NS}$ \\
FEV1 (L) & $2.62 \pm 0.55$ & $2.71 \pm 0.49$ & NS \\
\hline
\end{tabular}

NS: $P$ value $>0.05$.

In the IGC group, there were 8 cases of hypoglycemia; among the control group, there were 4 cases of hypoglycemia. The difference between the two groups is statistically significant. Hypokalemia occurred in 18 cases in the IGC group, whereas the number of cases with hypokalemia in the control group was 23 . The difference between the two groups is also statistically significant regarding hypokalemia. These data indicate that there were more cases of hypoglycemia and hypokalemia in the IGC group than the control group. (See Table 2 for the details.)

The median length of the hospital stay was 15 days in the IGC group, whereas in control group the median was 12 days. The difference between the two groups is statistically significant $(\mathrm{P}=0.05)$. This indicates that the patients in the IGC group had longer hospital stays than those in the control group. The median medical cost of the hospital stay was 8,866 Yuan in the IGC group, whereas in the control group, the median cost was 8,051 Yuan. However, the difference between the two groups is not statistically significant. The mortality and positive sputum culture rates were $4.3 \%, 4.4 \%$ and $21.74,22.73$ in the IGC group and the control group, respectively. No significant differences were found between the two groups in the mortality and positive sputum culture rates (See Table 2).
Table 2

Efficacy and safety of intensive glucose control

\begin{tabular}{lccc}
\hline & $\begin{array}{c}\text { Intensive glucose } \\
\text { control group } \\
(\mathbf{n}=\mathbf{6 9})\end{array}$ & $\begin{array}{c}\text { Control } \\
\text { group } \\
(\mathbf{n}=\mathbf{1 9 8})\end{array}$ & P value \\
\hline $\begin{array}{l}\text { Length of hospital stay (days) } \\
\text { Medical cost of hospital stay }\end{array}$ & $15(9,21)$ & $12(9,16)$ & 0.05 \\
$\left(\begin{array}{l}\text { Yuan) } \\
\text { Mortality (yes/no) }\end{array}\right.$ & $8866(5456,11063)$ & $8051(5511,10806)$ & 0.774 \\
Sputum culture (+/-) & $3 / 66$ & $9 / 189$ & 1.00 \\
Hypoglycemia (yes/no) & $15 / 54$ & $45 / 153$ & 0.86 \\
Hypokalemia (yes/no) & $8 / 61$ & $4 / 195$ & 0.003 \\
\hline
\end{tabular}

* 1 Yuan is equal approximately $0.16 \$$.

\section{Discussion}

In this prospective study, we found that intensive glucose control treatments are associated with adverse clinical outcomes in those patients admitted to the general ward with a physician diagnosis of AECOPD. The patients who underwent intensive glucose control treatments were more likely to have a longer than median stay or to incur higher expenses in the hospital than those with a less rigorous blood glucose control regimen.

An increasing number of studies have focused on the relationship between hyperglycemia and COPD. Chakrabarti found that mechanical ventilation was used more frequently in patients with a comorbidity of COPD and hyperglycemia and that hyperglycemia could affect the prognosis in patients with COPD.3 Loukides found that approximately $14.7 \%$ of the AECOPD patients also had diabetes (11). Wu L found that advanced glycation end products and their receptor (RAGE) are increased in patients with COPD (12).

Acute hyperglycemia during exacerbations of COPD is associated with poor exacerbation outcomes (13). A retrospective study has shown that for each $1 \mathrm{mmol} / 1$ increase in plasma glucose the risk of death or a prolonged hospital stay during COPD exacerbations was increased by $15 \%$ (4). Another prospective study also shows that acute hyperglycemia in COPD patients is associated with NIV and the 'late failure' ( $>48 \mathrm{~h}$ ) of NIV after an initial success (11). Parappil and his colleagues found that the risk of death increases with prolonging the length of the hospital stay in patients with a comorbidity of AECOPD and diabetes (14). Archer reported a prospective, non-randomized, single-arm study of intensive glycemic control in COPD patients with acute exacerbations and hyperglycemia admitted to the acute medical unit. These authors only included 20 patients (10 females, mean age 71 years) and demonstrated that intensive glucose control can be achieved within the acute medical ward with a similar safety and efficacy to that achieved with patients admitted to an intensive care unit, and the control of blood glucose could potentially 
have some benefit in cases of COPD exacerbations (15). The present study reveals that the median length of the hospital stay was 15 days in the IGC group, which was longer than that in the control group (12 days). Furthermore, there was no statistically significant difference in the mortality rates in the IGC group (4.3\%) and the control group (4.4\%). This finding is inconsistent with the previous studies. Whether race, gender, the rate used as an endpoint or other causes resulted in the inconsistent conclusion regarding this difference in the present study should be further investigated.

The strengths of the present study include that it is a prospective study, and we reported the total medical cost. We added the economic data to the analyses in our study, which made it possible to determine whether the treatment was cost-effective. There was no statistically significant difference between the intensive glucose control group (8,866 RMB) and the control group $(8,051$ RMB) in the total costs of the hospital stay. This result indicates a trend toward increased medical costs for the hospital stay among the patients assigned to IGC group. Furthermore, among the patients in the IGC group, there were more patients with hypoglycemia and hypokalemia. This may have been caused by the lower nurse:patient ratio and the less intensive monitoring in the general ward.

To the best of our knowledge, the present study is the first prospective study to compare the difference in medical costs between IGC and conventional glucose control in a large sample of elderly people. However, certain limitations of our study should be mentioned. First, this study was not a randomized controlled trial, and the groups were determined according to the judgment of the physician. This process may have a selection bias. Second, we did not adjust for other potential confounders, such as socio-economic status, the disease severity and other comorbidities. However, our study included a relatively large cohort of subjects. Third, this study was conducted in a hospital in China. Therefore, the result might not be representative of the situations found in other countries, especially those countries that have different medical systems compared with China.

\section{Conclusions}

In summary, intensive glucose control did not result in any benefits in patients with AECOPD and hyperglycemia admitted to the general ward and may prolong the length of stay and increase the risk of hypoglycemia and hypokalemia. Additional studies should be considered to analyze the cost-effectiveness of intensive glucose control in a general ward among AECOPD patients with hyperglycemia.

\section{Summary at a Glance}

Hyperglycemia is associated with an increased risk of hospital-acquired pulmonary infection in Acute Exacerbation of Chronic Obstructive Pulmonary Disease (AECOPD) patients. intensive glucose control (IGC) treatment may prolong the length of hospital stay and increase the risk of hypoglycemia and hypokalemia in patients with AECOPD.

Acknowledgements: This research was supported in part by a grant from the Sichuan Health Government Agency, China (No.070317). The authors thank the staff of the Department of Sichuan Academy of Medical Sciences \& Sichuan Provincial People's Hospital and all the participants (as well as their legal proxies) for their great contributions. This research absence of conflict of interest. We especially thank Prof. Costabel in the Ruhrlandklinik of Essen University in Germany for his very good suggestions.

Author contributions: Hui Ren designed the study and performed the experiments, as Co-first author; Lingyun Gao and Qiu Kui Hao performed the experiments, analyzed the data, and wrote the manuscript.

Conflict of interest: This research absence of conflict of interest

Ethical standard: The study was conducted in accordance with the Declaration of Helsinki and was approved by the ethics committee of Sichuan Academy of Medical Sciences \& Sichuan Provincial People's Hospital.

\section{References}

1. Global Initiative for Chronic Obstructive Lung Disease (GOLD), 2011. Global Strategy for the Diagnosis, Management and Prevention of COPD. http:// www.goldcopd.org/. Accessed:1 May 2012.

2. Anthonisen NR, Manfreda J, Warren CP, Hershfield ES, Harding GK, Nelson NA. Antibiotic therapy in exacerbations of chronic obstructive pulmonary disease. Ann. Intern. Med. 1987; 106: 196-204.

3. Chakrabarti B, Angus RM, Agarwal S, Lane S, Calverley PM. Hyperglycaemia as a predictor of outcome during non-invasive ventilation in decompensated COPD. Thorax 2009; 64: 857-62.

4. Baker EH, Janaway CH, Philips BJ, Brennan AL, Baines DL, Wood DM, Jones PW. Hyperglycaemia is associated with poor outcomes in patients admitted to hospital with acute exacerbations of chronic obstructive pulmonary disease. Thorax 2006; 61: 284-9.

5. Moretti M, Cilione C, Tampieri A, Fracchia C, Marchioni A, Nava S. Incidence and causes of non-invasive mechanical ventilation failure after initial success. Thorax 2000; 55: 819-25.

6. Esposito K, Nappo F, Marfella R, Giugliano G, Giugliano F, Ciotola M, Quagliaro L, Ceriello A, Giugliano D. Inflammatory cytokine concentrations are acutely increased by hyperglycemia in humans: role of oxidative stress. Circulation 2002; 106: 2067-72.

7. Russell ST, Rajani S, Dhadda RS, Tisdale MJ. Mechanism of induction of muscle protein loss by hyperglycaemia. Exp. Cell Res. 2009; 315: 16-25.

8. Capes SE, Hunt D, Malmberg K, Pathak P, Gerstein HC. Stress hyperglycemia and prognosis of stroke in nondiabetic and diabetic patients: a systematic overview. Stroke 2001; 32: 2426-32.

9. Baker EH, Wood DM, Brennan AL, Clark N, Baines DL, Philips BJ. Hyperglycaemia and pulmonary infection. Proc. Nutr. Soc. 2006; 65: 227-35.

10. Association CRM. Guideline for the diagnosis and treatment of chronic obstructive pulmonary disease (Chinese). Chinese Journal of Internal Medicine 2007; 46: 254-61.

11. Loukides S, Polyzogopoulos D. The effect of diabetes mellitus on the outcome of patients with chronic obstructive pulmonary disease exacerbated due to respiratory infections. Respiration 1996; 63: 170-3.

12. Wu L, Ma L, Nicholson LF, Black PN. Advanced glycation end products and its receptor (RAGE) are increased in patients with COPD. Respir. Med. 2011; 105: 329-36.

13. Baker EH, Bell D. Blood glucose: of emerging importance in COPD exacerbations. Thorax 2009; 64: 830-2.

14. Parappil A, Depczynski B, Collett P, Marks GB. Effect of comorbid diabetes on length of stay and risk of death in patients admitted with acute exacerbations of COPD. Respirology 2010; 15: 918-22.

15. Archer JR, Misra S, Simmgen M, Jones PW, Baker EH. Phase II study of tight glycaemic control in COPD patients with exacerbations admitted to the acute medical unit. BMJ Open 2011; 1: e000210. 